OPEN ACCESS

Edited by:

Yinduo Ji,

University of Minnesota Twin Cities,

United States

Reviewed by:

Esther Orozco,

Centro de Investigación y de Estudios Avanzados del Instituto Politécnico Nacional (CINVESTAV-IPN), Mexico

Víctor Antonio García-Angulo,

Universidad de Chile, Chile

*Correspondence:

Dongsheng Zhou

dongshengzhou1977@gmail.com

Xinxiang Huang

huxinx@ujs.edu.cn

Received: 22 April 2018

Accepted: 30 July 2018

Published: 05 September 2018

Citation:

Zhang Y, Hu L, Osei-Adjei G, Zhang Y, Yang $W$, Yin $Z$, Lu R, Sheng $X$, Yang $R$,

Huang $X$ and Zhou D (2018)

Autoregulation of ToxR and lts

Regulatory Actions on Major Virulence Gene Loci in Vibrio parahaemolyticus.

Front. Cell. Infect. Microbiol. 8:291.

doi: 10.3389/fcimb.2018.00291

\section{Autoregulation of ToxR and Its Regulatory Actions on Major Virulence Gene Loci in Vibrio parahaemolyticus}

\author{
Yiquan Zhang ${ }^{1}$, Lingfei Hu ${ }^{2}$, George Osei-Adjei ${ }^{1}$, Ying Zhang ${ }^{1}$, Wenhui Yang ${ }^{2}$, Zhe Yin ${ }^{2}$, \\ Renyun Lu ${ }^{1}$, Xiumei Sheng ${ }^{1}$, Ruifu Yang ${ }^{2}$, Xinxiang Huang ${ }^{1 *}$ and Dongsheng Zhou ${ }^{2 *}$ \\ ${ }^{1}$ School of Medicine, Jiangsu University, Zhenjiang, China, ${ }^{2}$ State Key Laboratory of Pathogen and Biosecurity, Beijing \\ Institute of Microbiology and Epidemiology, Beijing, China
}

Vibrio parahaemolyticus, the leading causative agent of seafood-associated gastroenteritis, harbors two major virulence gene loci T3SS1 and Vp-PAI (T3SS2 and tdh2). ToxR is a virulence regulator of vibrios. Cell density-dependent transcriptional pattern of toxR and its regulatory actions on T3SS1 and Vp-PAl have been previously reported, but the detailed regulatory mechanisms are still obscure. In the present work, we showed that the highest transcription level of toxR occurs at an $\mathrm{OD}_{600}=0.2-0.4$, which may be due to the subtle repression of ToxR and the quorum-sensing (QS) master regulator AphA. We also showed that ToxR is involved in regulating the mouse lethality, enterotoxicity, cytotoxicity, and hemolytic activity of $V$. parahaemolyticus. ToxR binds to the multiple promoter-proximal DNA regions within the T3SS1 locus to repress their transcription. In addition, ToxR occupies the multiple promoter-proximal DNA regions of $\mathrm{Vp}-\mathrm{PAl}$ locus to activate their transcription. Thus, ToxR regulates the multiple virulence phenotypes via directly acting on the T3SS1 and Vp-PAI genes. Data presented here provide a deeper understanding of the regulatory patterns of ToxR in V. parahaemolyticus.

\section{Keywords: Vibrio parahaemolyticus, ToxR, T3SS1, Vp-PAI, virulence}

\section{INTRODUCTION}

Vibrio parahaemolyticus is a Gram-negative halophilic bacterium that is highly abundant in estuarine and marine environments (Thompson et al., 2004). It is a well-known seafood-borne human pathogen that can cause acute gastroenteritis via consumption of raw or poorly cooked, contaminated seafood (Thompson et al., 2004). The major clinical symptoms include chills, fever, nausea, vomiting, watery diarrhea, and abdominal cramps (Broberg et al., 2011). Several large-scale outbreaks of $V$. parahaemolyticus infections have occurred since 1996, which are associated with the O3:K6 serovar and its serovariants (Yeung and Boor, 2004; Nair et al., 2007). Strains of the serotype O3:K6 and its genetic variants were known as the pandemic group of $V$. parahaemolyticus with higher levels of virulence relative to other groups (Yeung and Boor, 2004; Nair et al., 2007).

The pandemic $V$. parahaemolyticus strain RIMD2210633 expresses multiple virulence determinants including major ones, such as thermostable direct hemolysin (TDH) and type III secretion system 1 (T3SS1) and system 2 (T3SS2) (Makino et al., 2003). The TDH can cause 
$\beta$-type hemolysis when the bacterium is grown on the special Wagatsuma agar, a phenomenon known as the Kanagawa phenomenon (KP) (Miyamoto et al., 1969; Honda et al., 1988). The TDH is also required for the lethality in mice and partially contributes to the cytotoxicity and enterotoxicity of V. parahaemolyticus (Raimondi et al., 2000; Naim et al., 2001; Park et al., 2004a; Hiyoshi et al., 2010). The strain harbors two copies of $t d h$ ( $t d h 2$ :VPA1314 and $t d h 1$ :VPA1378) on the smaller chromosome II (Makino et al., 2003). However, the production of $t d h 2$ is predominantly responsible for the TDH activities because of much higher transcriptional levels of $t d h 2$ than that of $t d h 1$ (Nishibuchi and Kaper, 1990; Okuda and Nishibuchi, 1998). The two copies of $t d h$ genes together with T3SS2 (VPA13201370) locus are located on the $80 \mathrm{~kb}$ pathogenicity island termed Vp-PAI (VPA1312-1398) (Makino et al., 2003). The T3SS1 mainly contributes to the cytotoxicity of $V$. parahaemolyticus that induces a series of events including autophagy, membrane blebbing, and, lastly, cell lysis (Park et al., 2004b; Hiyoshi et al., 2010; Letchumanan et al., 2014). By contrast, T3SS2 has been demonstrated to be involved in enterotoxicity of $V$. parahaemolyticus that causes the accumulation of intestinal fluid in a rabbit ileal loop model (Park et al., 2004b; Hiyoshi et al., 2010). The genetic organizations of both T3SS1 (VP1656VP1702) and T3SS2 are complex gene clusters that are composed of more than 40 consecutive genes, respectively (Makino et al., 2003).

The ToxR is a membrane-localized regulatory protein that plays an essential role in modulating bacterial persistence and virulence (Crawford et al., 2003; Childers and Klose, 2007). V. cholerae ToxR binds to the promoter-proximal DNA region of toxT to enhance its transcription, and ToxT, in turn, activates the virulence genes including $c t x$ and tcp encoding toxin coregulated pilus (TCP) and cholera toxin (CT), respectively (Dirita et al., 1991; Higgins and Dirita, 1994). The ToxR also can directly activate $c t x$ transcription in a ToxT-independent manner in the presence of bile acids (Hung and Mekalanos, 2005). Additionally, some outer membrane proteins (Omps), such as ompU and $o m p T$, as well as biofilm formation are also under the control of ToxR, suggesting that ToxR is necessary for the survival of the bacteria under adverse environmental stimuli (Provenzano and Klose, 2000; Provenzano et al., 2001; Valeru et al., 2012). $V$. parahaemolyticus ToxR is highly similar to $V$. cholerae ToxR (Lin et al., 1993). It has been demonstrated that ToxR is involved in regulating the expression of TDH, T3SS1 as well as T3SS2 genes in V. parahaemolyticus (Lin et al., 1993; Whitaker et al., 2012; Hubbard et al., 2016). A previous study showed that ToxR represses the transcription of T3SS1 genes most likely via direct activation of CalR, which is a direct repressor of T3SS1 (OseiAdjei et al., 2017). However, whether ToxR has direct regulatory effects on T3SS1 genes and the detailed regulatory actions of ToxR on $t d h$ and T3SS2 genes are still obscure. In addition, cell density-dependent transcriptional patterns of tox $R$ have been observed in V. cholerae and V. parahaemolyticus (Xu et al., 2010; Zhang et al., 2017), which suggest a possible connection between ToxR expression and quorum sensing (QS) in pathogenic vibrios. However, the molecular mechanisms also need to be further investigated.
The QS is a cell-cell signaling process that bacteria use to modulate communal behavior and gene regulation in response to cell density changes and the presence of chemical molecules termed autoinducers (AIs) in the surroundings (Srivastava and Waters, 2012). The QS controls gene expression generally via the downstream master regulators (Ng and Bassler, 2009). The AphA and OpaR represent the two master regulators of QS in $V$. parahaemolyticus that function at low cell density (LCD) and high cell density (HCD), respectively (Sun et al., 2012; Zhang et al., 2012). In the present work, we showed that AphA indirectly represses the transcription of toxR at LCD, whereas OpaR has no regulatory actions on toxR transcription. Meanwhile, ToxR also shows no regulatory actions on both $a p h A$ and $o p a R$. However, autorepression of ToxR was observed herein. In addition, ToxR acts as a virulence regulator of $V$. parahaemolyticus via direct repression of T3SS1 genes, but activates Vp-PAI (T3SS2 and $t d h 2$ ) genes. This work promotes us to gain a deeper understanding of the regulatory patterns of ToxR in $V$. parahaemolyticus.

\section{MATERIALS AND METHODS}

\section{Bacterial Strains and Growth Conditions}

V. parahaemolyticus RIMD2210633 was used as the wild-type (WT) strain in the present work (Makino et al., 2003). The nonpolar $a p h A, o p a R$, and toxR single-gene deletion mutants derived from WT (designated as $\triangle a p h A, \triangle o p a R$, and $\triangle$ toxR, respectively), and the corresponding complementary mutants were previously described (Sun et al., 2012; Zhang et al., 2012, 2017). All the primers used are listed in Table 1.

The 2.5\% Bacto heart infusion (HI) broth (BD Bioscience, USA) was used to culture V. parahaemolyticus strains. Overnight bacterial cell cultures were diluted 1:50 into $15 \mathrm{ml}$ of fresh $\mathrm{HI}$ broth, and allowed to grow at $37^{\circ} \mathrm{C}$ with shaking at $200 \mathrm{rpm}$ to reach $\mathrm{OD}_{600} \approx 1.0$ (the midexponential growth phase), and then diluted 1:1000 into $15 \mathrm{ml}$ of fresh $\mathrm{HI}$ broth and allowed to grow under the same conditions to reach the required cell densities. When necessary, the medium was supplemented with $50 \mu \mathrm{g} / \mathrm{ml}$ gentamicin, $5 \mu \mathrm{g} / \mathrm{ml}$ chloramphenicol, or $0.1 \%$ arabinose.

\section{Murine Infection Assay}

The murine infection assay was done as previously described (Sun et al., 2014). Briefly, $0.1 \mathrm{ml}$ of the $10^{8} \mathrm{CFU} / \mathrm{ml}$ bacterial suspensions in phosphate buffered saline (PBS) (pH7.2) was inoculated intraperitoneally into each of the 15 female BALB/c mice that were 25 to 28 days old, after which the number of mice killed at specified times was monitored daily. The PBS was used as a control in one trial to confirm experimental outcomes.

\section{Kanagawa Phenomenon (KP) Test}

The KP test was done as previously described (Honda et al., 1980). Briefly, $5 \mu \mathrm{l}$ of the third-round cell cultures were inoculated onto Wagatsuma agar medium containing $5 \%$ rabbit red blood cells (RBCs), $5 \mu \mathrm{g} / \mathrm{ml}$ chloramphenicol, and $0.1 \%$ arabinose. The radius from the point of inoculation to the edge of the $\beta$-hemolysin zone was measured after static incubation at $37^{\circ} \mathrm{C}$ for at least $24 \mathrm{~h}$. 
TABLE 1 | Oligonucleotide primers used in this study.

\begin{tabular}{|c|c|}
\hline Target & Primers (forward/reverse, $5^{\prime}-3^{\prime}$ ) \\
\hline \multicolumn{2}{|c|}{ CONSTRUCTION OF MUTANTS } \\
\hline \multirow[t]{3}{*}{ opaR } & $\begin{array}{l}\text { GTGACTGCAGACTGCCTTGGTAACGCTCTG } \\
\text { /GTTCGTGTTCAAATCTGAGCTATCCATITCCTTGCCATTTG }\end{array}$ \\
\hline & $\begin{array}{l}\text { CAAATGGCAAGGAAAATGGATAGCTCAGATTTGAACACGAAC } \\
\text { /GTGAGCATGCATGGGCTGCATCAGGTCG }\end{array}$ \\
\hline & $\begin{array}{l}\text { GTGACTGCAGACTGCCTTGGTAACGCTCTG } \\
\text { /GTGAGCATGCATGGGCTGCATCAGGTCG }\end{array}$ \\
\hline \multirow[t]{3}{*}{ aphA } & $\begin{array}{l}\text { GTGACTGCAGCGCAGCAAATAACCAGAC } \\
\text { /CCAATCACTTCAAGTTCTGTTGTCTTCAATCCAAATGGTC }\end{array}$ \\
\hline & $\begin{array}{l}\text { GACCATTGGATTGAAGACAACAGAACTTGAAGTGATTGG } \\
\text { /GTGAGCATGCGTITCGTGACCGCTGTG }\end{array}$ \\
\hline & $\begin{array}{l}\text { GTGACTGCAGCGCAGCAAATAACCAGAC } \\
\text { /GTGAGCATGCGTITCGTGACCGCTGTG }\end{array}$ \\
\hline \multirow[t]{3}{*}{ tox $R$} & $\begin{array}{l}\text { GTGACTGCAGAAACGCAATTTGTCTGATG } \\
\text { /ATCTTCATGCTGGCCTCCTTIAGTTCTTCTTAGATGGATGATG }\end{array}$ \\
\hline & $\begin{array}{l}\text { CATCATCCATCTAAGAAGAACTAAAGGAGGCCAGCATGAAGAT } \\
\text { /GTGAGCATGCAATTCGGCGGCTTGTTC }\end{array}$ \\
\hline & $\begin{array}{l}\text { GTGACTGCAGAAACGCAATTTGTCTGATG } \\
\text { /GTGAGCATGCAATTCGGCGGCTITGTTC }\end{array}$ \\
\hline \multicolumn{2}{|c|}{ CONSTRUCTION OF COMPLEMENTED MUTANTS } \\
\hline toxR & $\begin{array}{l}\text { GATTCTAGAAGGAGGAATTCACCATGACTAACATCGGCACCAA } \\
\text { /GACAAGCTITATTTGCAGATGTCTGTTGG }\end{array}$ \\
\hline \multicolumn{2}{|c|}{ PROTEIN EXPRESSION } \\
\hline opaR & $\begin{array}{l}\text { AGCGGGATCCATGGACTCAATTGCAAAGAG } \\
\text { /AGCGAAGCTITAGTGTTCGCGATTGTAG }\end{array}$ \\
\hline aphA & $\begin{array}{l}\text { AGCGGGATCCATGTCATTACCACACGTAATC } \\
\text { /AGCGAAGCTITAACCAATCACTTCAAGTTC }\end{array}$ \\
\hline toxR & $\begin{array}{l}\text { AGCGGGATCCATGACTAACATCGGCACCAA } \\
\text { /AGCGAAGCTITIAAGGATTCACAGCAGAAG }\end{array}$ \\
\hline $\begin{array}{l}\text { qPCR } \\
\text { aphA }\end{array}$ & AGCATCGGTTACTTCTGGAAAG/GTTGAACAGCACAAGCCATAAG \\
\hline opaR & TGTCTACCAACCGCACTAACC/GCTCTTTCAACTCGGCTTCAC \\
\hline toxR & TTGTTGGCGTGAGCAAGG/TAGCAGAGGCGTCATTGTTATC \\
\hline exsB & ATGAAAAGCAGTAAGTGGGC/CTGAGAAGCAACAGTAAGAC \\
\hline VP1687 & TGCTCACCGTTGCCAAATAG/GCGACGCTTTCATGTATTGC \\
\hline $\operatorname{vop} N$ & GGAATGGATTGGAATCGTC/CCACCGTCTITATITGC \\
\hline vtrA & AGTCTAGGCTCACAAGATCG/AAATGGGCTCTGATGTTACG \\
\hline vopB2 & ACCAGCCTCAGCAACAAGC/CTTTCACGAATACTACGC \\
\hline tdh2 & ATGTAAAAAGAAAACCGTACA/AACACAGCAGAATGACCGTG \\
\hline \multicolumn{2}{|c|}{ PRIMER EXTENSION } \\
\hline aphA & /GCTCTTACTGGCGCTTGAG \\
\hline opaR & /ATCCATTTCCTTGCCATTTG \\
\hline toxR & /TTAGTTCTTCTTAGATGGATGATG \\
\hline exs $B$ & /GTCTTATTATGATTIATIITACAC \\
\hline VP1687 & /GGCAACGGTGAGCAAAATC \\
\hline $\operatorname{vop} N$ & /GACGATTCCAATCCATTCCG \\
\hline vtrA & /CCGCTATCGCTGCTATTT \\
\hline vopB2 & /GAGATTCGTAGCGTATAAGTGC \\
\hline tdh2 & /GCAAAATATCGGTACTTCA \\
\hline \multicolumn{2}{|c|}{ LacZ FUSION } \\
\hline aphA & $\begin{array}{l}\text { GCGCGTCGACCATTCGTAATACAAAAGG } \\
\text { /GCGCGGTACCTTCCAGAAGTAACCGATGCTAG }\end{array}$ \\
\hline opaR & $\begin{array}{l}\text { GCGCGTCGACTCCATCGTGTTGCCGTAGC } \\
\text { /GCGCGGTACCCAATATCTGCGTGACCACCAC }\end{array}$ \\
\hline
\end{tabular}

(Continued)
TABLE 1 | Continued

\begin{tabular}{|c|c|}
\hline Target & Primers (forward/reverse, $5^{\prime}-3^{\prime}$ ) \\
\hline toxR & $\begin{array}{l}\text { GCGCGTCGACATCGTTAAGGTATTTGCA } \\
\text { /GCGCGAATTCCGAGCGAATTACTATTTGG }\end{array}$ \\
\hline exsB & $\begin{array}{l}\text { ATATGTCGACATTGTCCGTCAAATGCAGTTC } \\
\text { /TITGAATTCCATATACATTCGCTTGGCTCTG }\end{array}$ \\
\hline VP1687 & $\begin{array}{l}\text { GCGCGTCGACGCATTATTGACGCCAGTATCG } \\
\text { /GCGCTCTAGAGGCAACGGTGAGCAAAATC }\end{array}$ \\
\hline vopN & $\begin{array}{l}\text { GCGGTCGACCAGATTGCTGAATATCGGTG } \\
\text { /GCGTCTAGAAAGCGATTGAGTGGCGTTG }\end{array}$ \\
\hline vtrA & $\begin{array}{l}\text { GCGCGTCGACTACGCTTCCAATAATCACC } \\
\text { /GCGCGAATTCCCGATCTTGTGAGCCTAGA }\end{array}$ \\
\hline vopB2 & $\begin{array}{l}\text { GCGGTCGACGCGTACTAAGTGATGAAGAG } \\
\text { /GCGTCTAGACAACAGAACCACTITCAGC }\end{array}$ \\
\hline tdh2 & $\begin{array}{l}\text { GCGCGTCGACAATTCACGACGAATCGGAG } \\
\text { /GCGCGAATTCATATCGGTACTTCATAAA }\end{array}$ \\
\hline \multicolumn{2}{|l|}{ EMSA } \\
\hline aphA & CATTCGTAATACAAAAGG/TTCCAGAAGTAACCGATGCTAG \\
\hline opaR & TCCATCGTGTTGCCGTAGC/CAATATCTGCGTGACCACCAC \\
\hline toxR & ATCGTTAAGGTATTTGCA/CGAGCGAATTACTATTTGG \\
\hline exs $B$ & АTTGTCCGTCAAATGCAGTTC/CATATACATTCGCTTGGCTCTG \\
\hline VP1687 & GCATTATTGACGCCAGTATCG/GGCAACGGTGAGCAAAATC \\
\hline vopN & CAGATTGCTGAATATCGGTG/AAGCGATTGAGTGGCGTTG \\
\hline vtrA & TACGCTTCCAATAATCACC/CCGATCTTGTGAGCCTAGA \\
\hline vopB2 & GCGTACTAAGTGATGAAGAG/CAACAGAACCACTTTCAGC \\
\hline tdh2 & AATTCACGACGAATCGGAG/ATATCGGTACTTCATAAA \\
\hline \multicolumn{2}{|c|}{ DNase I FOOTPRINTING } \\
\hline toxR & TाTAGGGACGACTTTGTG/TTAGTTCTTCTTAGATGGATGATG \\
\hline VP1687 & CACCAGAGTAGGGCATCAC/CAGAGTGACCCAGAGCCG \\
\hline $\operatorname{vopN}$ & CAGATTGCTGAATATCGGTG/ATTGATAATACTCATTCACTTGC \\
\hline$v \operatorname{tr} A$ & СATTGCCCAAGTTTATCAG/CCGATCTTGTGAGCCTAGAC \\
\hline tdh2 & TCATTACCACAACGCCTCTG/CTGTGATTCCTCAAGCGG \\
\hline
\end{tabular}

\section{Cytotoxicity Assay}

The cytotoxicity assay was performed as previously described (Hiyoshi et al., 2010; Sun et al., 2014). Briefly, the third-round cell cultures were washed and serially diluted with the prewarmed Dulbecco's modified Eagle's medium (DMEM) lacking phenol red for CFU measurement and infection. HeLa cells were infected with $10^{6} \mathrm{CFU}$ of bacteria for $3 \mathrm{~h}$ at a multiplicity of infection (MOI) of 2.5. Subsequently, the release of lactate dehydrogenase (LDH) into the medium was quantified with a CytoTox $96^{\circledR}$ Nonradioactive Cytotoxicity Assay kit (Promega, USA) according to the manufacturer's instructions.

\section{Rabbit Ileal Loop Test}

The rabbit ileal loop test was done as previously described (Nishibuchi et al., 1992; Sun et al., 2014). Briefly, four loops $(10 \mathrm{~cm}$ each in length) were placed in the small intestine of each of the six rabbits. One milliliter of bacterial suspension $\left(10^{9} \mathrm{CFU} / \mathrm{ml}\right)$ was injected into each ileal loop. The rabbits were sacrificed by the venous air embolism $14 \mathrm{~h}$ postinjection, and the fluid accumulation was calculated as the amount of accumulated fluid of each ligated rabbit ileal loop. Isoflurane was employed for the inhalation anesthesia of rabbits before each surgery. All 
the animal experiments were approved by the Committee on Animal Research of the Academy of Military Medical Sciences and carried out per the approved guideline.

\section{Quantitative PCR (qPCR)}

Total RNAs were extracted from $V$. parahaemolyticus strains using the TRIzol Reagent (Invitrogen, USA). The contaminating genomic DNA in the total RNAs was removed using the Ambion's DNA-free ${ }^{\mathrm{TM}}$ Kit according to the manufacturer's instructions. The cDNAs were generated by using $8 \mu \mathrm{g}$ of total RNAs and $3 \mu \mathrm{g}$ of random hexamer primers. The SYBR Green qPCR assay was performed and analyzed as previously described (Gao et al., 2011). The relative mRNA levels of each target gene were determined based on the standard curve of 16S rRNA (reference gene) expression for each RNA preparation.

\section{Primer Extension Assay}

The primer extension assay was done as previously described (Gao et al., 2011; Zhang et al., 2012). Briefly, about $10 \mu \mathrm{g}$ of total RNAs were annealed with 1 pmol of $5^{\prime}-{ }^{32} \mathrm{P}$-end labeled reverse oligonucleotide primer to generate $\mathrm{cDNAs}$ using a Primer Extension System (Promega, USA). The same labeled primer was used for sequencing with the AccuPower and Top DNA Sequencing Kit (Bioneer, Korea). The primer extension products and sequencing materials were concentrated and analyzed in an $8 \mathrm{M}$ urea-6\% polyacrylamide gel electrophoresis, and the results were detected by autoradiography with the Fuji Medical X-ray film (Fuji Photo Film Co., Ltd. Japan).

\section{LacZ Fusion and $\beta$-Galactosidase Assay}

For the LacZ fusion assay (Gao et al., 2011; Sun et al., 2012), the regulatory DNA region of each indicated gene was cloned into the corresponding restriction endonuclease sites of the pHRP309 plasmid harboring a promoterless lacZ reporter gene and a gentamicin-resistance gene (Parales and Harwood, 1993). The resultant plasmid was then transferred into $V$. parahaemolyticus strains, in which the promoterless lac $Z$ gene can be expressed under the control of the target promoters. Thus, the regulatory actions of ToxR on target genes can be assessed by measuring the $\beta$-galactosidase activities in cellular extracts of WT and $\Delta$ toxR ( $\beta$-Galactosidase Enzyme Assay System, Promega).

\section{Preparation of $6 x$ His-Tagged Proteins}

The entire coding regions of $a p h A, o p a R$, and the truncated toxR (1-528 bp, a.a.1-176) were cloned into plasmid pET28a (Novagen, USA), respectively. The recombinant plasmids encoding His-

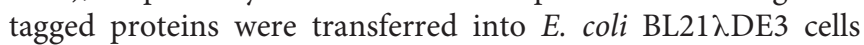
for protein expression (Kleber-Janke and Becker, 2000). The conditions for expression and purification of His-tagged AphA, OpaR, and ToxR have been described previously (Sun et al., 2012; Zhang et al., 2012, 2017).

\section{Electrophoretic Mobility Shift Assay (EMSA)}

The EMSA was done as previously described (Sun et al., 2012; Zhang et al., 2012, 2017). Briefly, the $5^{\prime}$-ends of the promoterproximal DNA region of each target gene were labeled using $\left[\gamma^{-32} \mathrm{P}\right]$ ATP and T4 polynucleotide kinase. DNA binding was performed in a $10 \mu \mathrm{l}$ reaction volume containing binding buffer ( $1 \mathrm{mM} \mathrm{MgCl}, 0.5 \mathrm{mM}$ EDTA, $0.5 \mathrm{mM}$ DTT, $50 \mathrm{mM}$ $\mathrm{NaCl}, 10 \mathrm{mM}$ Tris- $\mathrm{HCl} / \mathrm{pH} 7.5$, and $10 \mathrm{mg} / \mathrm{ml}$ salmon sperm DNA), labeled DNA probe (about 2,000 CPM/ $\mu \mathrm{l}$ ), and increasing amounts of His-tagged protein. After being incubated at room temperature for $30 \mathrm{~min}$, the products were loaded onto a native $4 \%(\mathrm{w} / \mathrm{v})$ polyacrylamide gel, and electrophoresed in $0.5 \times \mathrm{TBE}$ buffer for about $50 \mathrm{~min}$ at $200 \mathrm{~V}$. Radioactive species were detected by autoradiography after exposure to Fuji Medical Xray film at $-20^{\circ} \mathrm{C}$. Three controls were included in each EMSA experiment: (1) cold probe as specific DNA competitor (the same promoter-proximal DNA region unlabeled), (2) negative probe as nonspecific DNA competitor (the unlabeled coding region of the $16 \mathrm{~S}$ rRNA gene), and (3) nonspecific protein competitor (rabbit anti-F1-protein polyclonal antibodies).

\section{DNase I Footprinting}

The DNase I footprinting was done as previously described (Sun et al., 2012; Zhang et al., 2012). Briefly, single strand ${ }^{32} \mathrm{P}-$ $5^{\prime}$ end-labeled probes were incubated with increasing amounts of His-tagged protein for $30 \mathrm{~min}$ at room temperature, in a final $10 \mu \mathrm{l}$ reaction volume containing the binding buffer used in EMSA. Before DNA digestion, $10 \mu \mathrm{l}$ of $\mathrm{Ca}^{2+} / \mathrm{Mg}^{2+}$ solution $\left(5 \mathrm{mM} \mathrm{CaCl}_{2}\right.$ and $10 \mathrm{mM} \mathrm{MgCl}_{2}$ ) was added, followed by incubation for $1 \mathrm{~min}$ at room temperature. The optimized RQ1 RNase-Free DNase I (Promega, USA) was then added to the reaction mixture and the mixture was incubated at room temperature for 40 to $90 \mathrm{~s}$. The reaction was quenched by adding $9 \mu \mathrm{l}$ of stop solution $(200 \mathrm{mM} \mathrm{NaCl}, 30 \mathrm{mM}$ EDTA, and $1 \% \mathrm{SDS}$ ), followed by incubation for $1 \mathrm{~min}$ at room temperature. The partially digested DNA samples were extracted with phenol/chloroform, precipitated with ethanol, and analyzed in $6 \%$ polyacrylamide/ $8 \mathrm{M}$ urea gel. Protected regions were identified by comparison with the sequence ladders. The templates for DNA sequencing were the same as the DNA fragments for DNase I footprinting assay. Radioactive species were detected by autoradiography after exposure to Fuji Medical $\mathrm{X}$-ray film at $-20^{\circ} \mathrm{C}$.

\section{Prediction of the Minimal ToxR Binding Sites Within Target Regulatory Regions}

The 500 bp upstream DNA regions of the genes tested (Table 2) were retrieved from the genome sequence of RIMD 2210633 with the "retrieve-seq" program (Van Helden, 2003). Subsequently, the DNA binding box of ToxR (Goss et al., 2013) was used to statistically predict the presence of ToxR box-like sequences within the target upstream DNA regions by using the matricespaster tool (Van Helden, 2003). This analysis generated the weight scores for each target upstream DNA region. The higher score values represented the higher probability of ToxR and the upstream DNA region association.

\section{Experimental Replicates and Statistical Methods}

The LacZ fusion and qPCR assays were performed with at least three independent bacterial cultures, and the values were 
TABLE 2 | Predicted ToxR box-like sequences within upstream DNA regions.

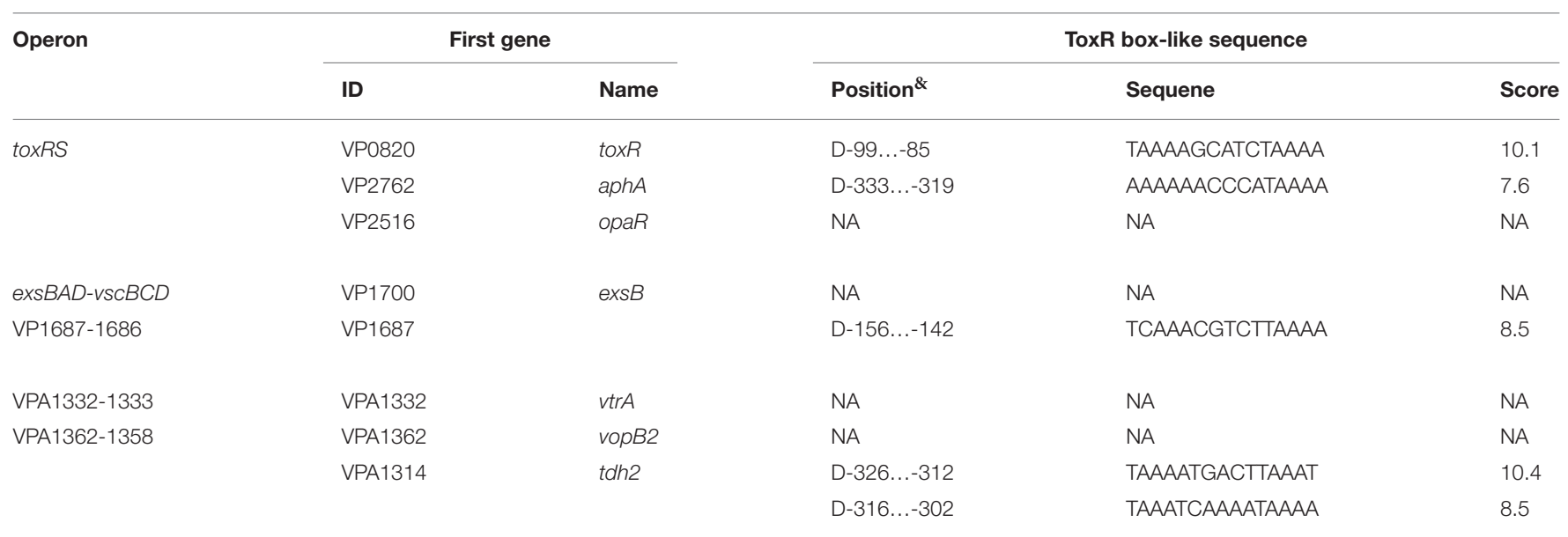

\&, "D" indicates the direct sequence while "R" the reverse one; minus numbers denote the nucleotide positions upstream of indicated genes; "NA" represents "not applicable."

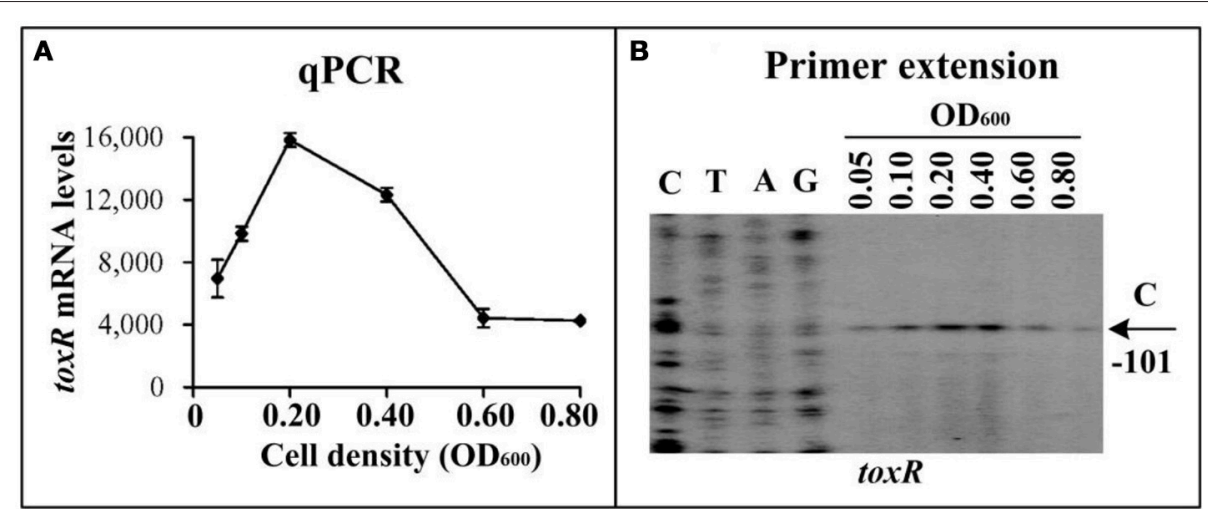

FIGURE 1 | Cell density-dependent transcription of toxR. The WT strain was grown in $\mathrm{HI}$ broth at $37^{\circ} \mathrm{C}$, and bacterial cells were harvested at various $\mathrm{OD}_{600}$ values. (A) qPCR. The relative mRNA level of each target gene was tested in WT at different cell densities. (B) primer extension. An oligonucleotide primer was designed to be complementary to the RNA transcript of toxR. The primer extension products were analyzed with an $8 \mathrm{M}$ urea- $6 \%$ acrylamide sequencing gel. Lanes $\mathrm{C}$, T, A, and G represented Sanger sequencing reactions. The transcriptional start sites were indicated by arrows with nucleotides and positions. The minus numbers under the arrows indicated the nucleotide positions upstream of start codon of toxR.

expressed as the mean \pm standard deviation (SD). Paired Student's $t$-test was used to calculate statistically significant differences, $p<0.01$ was considered to indicate statistical significance. The data for phenotype, primer extension, EMSA, and DNase I footprinting assays were done at least two independent times.

\section{RESULTS}

\section{Cell Density-Dependent Transcription and Autorepression of ToxR}

The qPCR and primer extension assays were employed to measure the transcription change of tox $R$ at the different growth phases of WT in HI broth (Figure 1). The results showed that the mRNA level of toxR increased considerably with the increase of cell density from an $\mathrm{OD}_{600}$ value of 0.05 to 0.2 , and the highest level occurred at an $\mathrm{OD}_{600}$ value of 0.2 to 0.4 , but it dramatically decreased when the $\mathrm{OD}_{600}$ value was higher than 0.4 (Figure 1).
These results suggested that transcription of toxR is very likely to be regulated by QS in V. parahaemolyticus. AphA and OpaR are the two bottom regulators of QS in $V$. parahaemolyticus operating at LCD and HCD, respectively (Sun et al., 2012; Zhang et al., 2012; Lu et al., 2018). Thus, bacterial cells were harvested at an $\mathrm{OD}_{600}$ value of 0.15 and 0.8 , respectively, to investigate the regulatory actions of AphA and OpaR on toxR transcription. The qPCR and primer extension results showed that the mRNA level of toxR increased in $\triangle a p h A$ relative to that in WT (Supplementary Figures 1A,B). The LacZ fusion assay further indicated that the promoter activity of tox $R$ was much higher in $\triangle a p h A$ than that in WT (Supplementary Figure 1C). The EMSA result showed that His-AphA was unable to bind to the upstream DNA fragment of toxR in a dose-dependent manner (Supplementary Figure 1D). However, His-AphA was able to bind in a dose-dependent manner to the upstream DNA regions of other targets such as aphA, qrr4, and opaR even at a much lower protein amount (Sun et al., 2012; Zhou et al., 2013). Thus, AphA indirectly repressed toxR transcription at LCD. By 
contrast, OpaR seems to have no regulatory action on toxR at HCD (Supplementary Figure 2). These results cannot explain the transcriptional patterns of toxR observed in Figure 1, so there must be an additional unknown regulator (s) that regulate toxR.

A ToxR box-like sequence (TAAAAGCATCTAAAA, also shown in Table 2) was detected within the promoter-proximal DNA region of toxR by using the DNA binding box of ToxR and the online matrix-scan tool (http://embnet.ccg.unam.mx/ rsat/), suggesting an autoregulation mechanism of ToxR in $V$. parahaemolyticus. Thus, the bacterial cells were harvested at an $\mathrm{OD}_{600}$ value of 0.4 to investigate the autoregulation of ToxR. Since the toxR coding region was deleted from the $V$. parahaemolyticus genome, we thus chose a reverse primer located upstream of tox $R$ but next to the start codon to conduct the primer extension assay (Table 1). As shown in Figure 2A, a single transcription start site located at $101 \mathrm{bp}$ upstream of toxR was detected, and its transcriptional activity was hugely enhanced in $\Delta$ tox $R$ relative to $\mathrm{WT}$. The recombinant lacZ fusion plasmid that contains the regulatory region of tox $R$ and a promoterless lac $Z$ gene was transferred into $\Delta$ tox $R$ and WT, respectively, to test the action of ToxR on its own promoter. The result showed a significantly enhanced promoter activity of tox $R$ in $\Delta$ toxR relative to WT (Figure 2B). In vitro EMSA results showed that His-ToxR could bind to its own regulatory region in a dosedependent manner, but it could not bind to the 16S rRNA gene as the negative control (Figure 2C). As further determined by DNase I footprinting assay (Figure 2D), His-ToxR protected a single DNA region upstream of toxR against DNase I digestion, which was considered as the ToxR site. Thus, ToxR represses its own gene transcription in a direct manner.

We further investigated the ToxR-mediated $a p h A$ and $o p a R$ transcription. The $\mathrm{qPCR}$, primer extension, and LacZ fusion assays showed that ToxR does not regulate the transcription of both $a p h A$ and $o p a R$ (Supplementary Figure 3). The EMSA results showed that His-ToxR was unable to specifically bind to the upstream DNA fragments of $a p h A$ and $o p a R$ in a dosedependent manner (Supplementary Figure 3).

Taken together, AphA indirectly represses toxR at LCD and autorepression of ToxR at the transition from LCD to HCD $\left(\mathrm{OD}_{600}=0.2-0.4\right)$ may result in the cell-density transcription pattern of toxR.

\section{Involvement of ToxR in Virulence}

The virulent activity of WT/pBAD33, $\Delta$ toxR/pBAD33, and $\Delta$ toxR/pBAD33-toxR (C- $\Delta$ tox $R$ ) were performed using the listed phenotypes. First, the survival rates of mice infected with WT, $\triangle$ toxR, and PBS (negative control) were determined, and the lethality in mice for $\triangle$ toxR significantly decreased relative to WT and PBS (Figure 3A). We did not use the strains carrying the pBAD33 or pBAD33-toxR plasmid because of the lack of arabinose in mice for efficiently inducing expression of pBAD33-toxR (Sun et al., 2014). Secondly, cytotoxicity against HeLa cells was investigated regarding the release of $\mathrm{LDH}$ from cultured cells (Figure 3B). The cytotoxicity of cells infected with $\Delta$ toxR/pBAD33 was hugely enhanced compared with that infected with WT/pBAD33 and C- $\Delta$ toxR. Thirdly, the hemolytic activity was measured by using $\mathrm{KP}$ test on the Wagatsuma

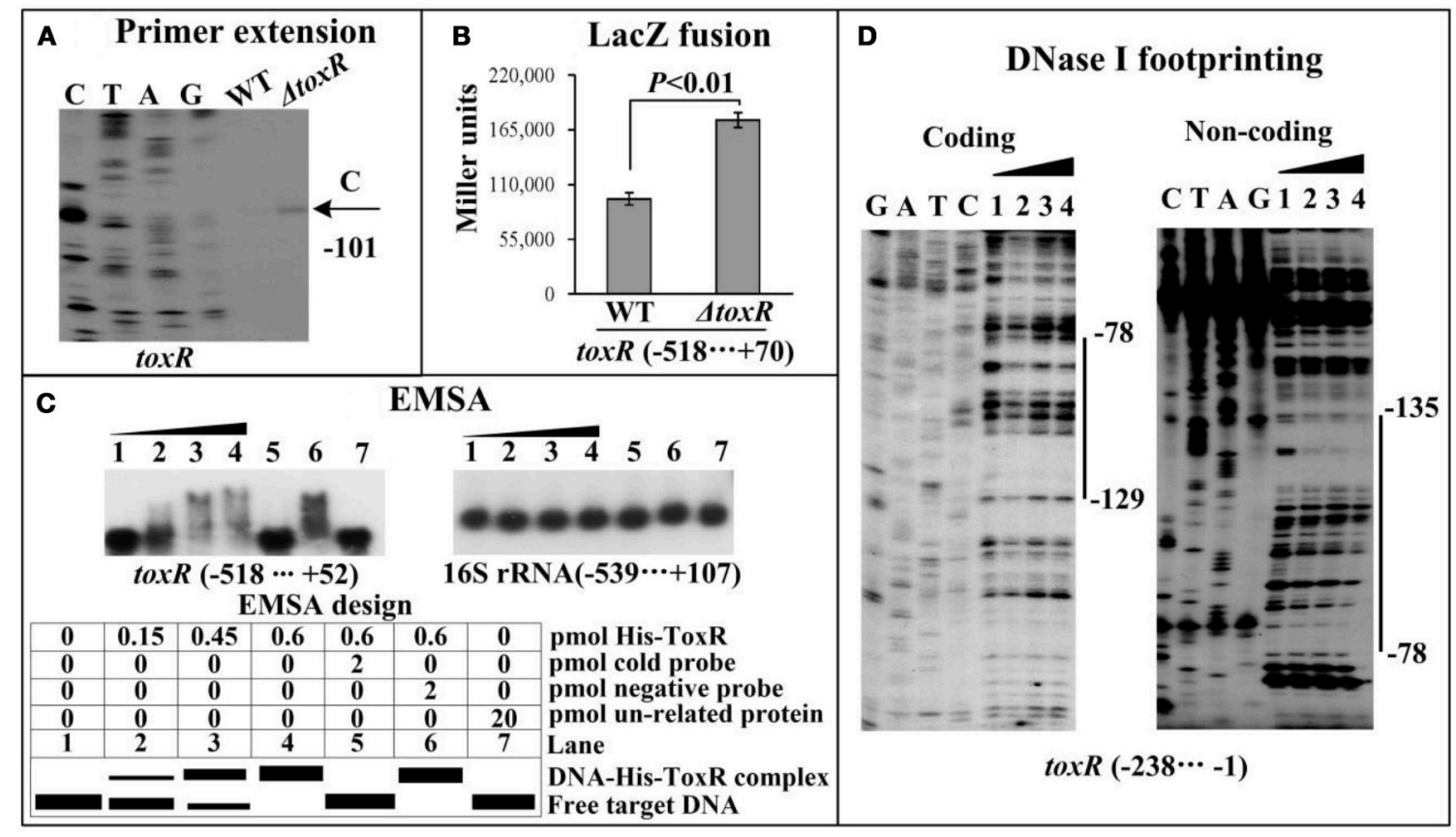

FIGURE 2 | Autoregulation of ToxR. The primer extension (A) was done as Figure 1, while the LacZ fusion (B) and EMSA (C) were done as Supplementary Figure 1. (D) DNase I footprinting. Labeled coding or noncoding DNA probes were incubated with increasing amounts of purified His-ToxR proteins, and then subjected to DNase I footprinting assay. The footprint regions were indicated by vertical bars with positions. 


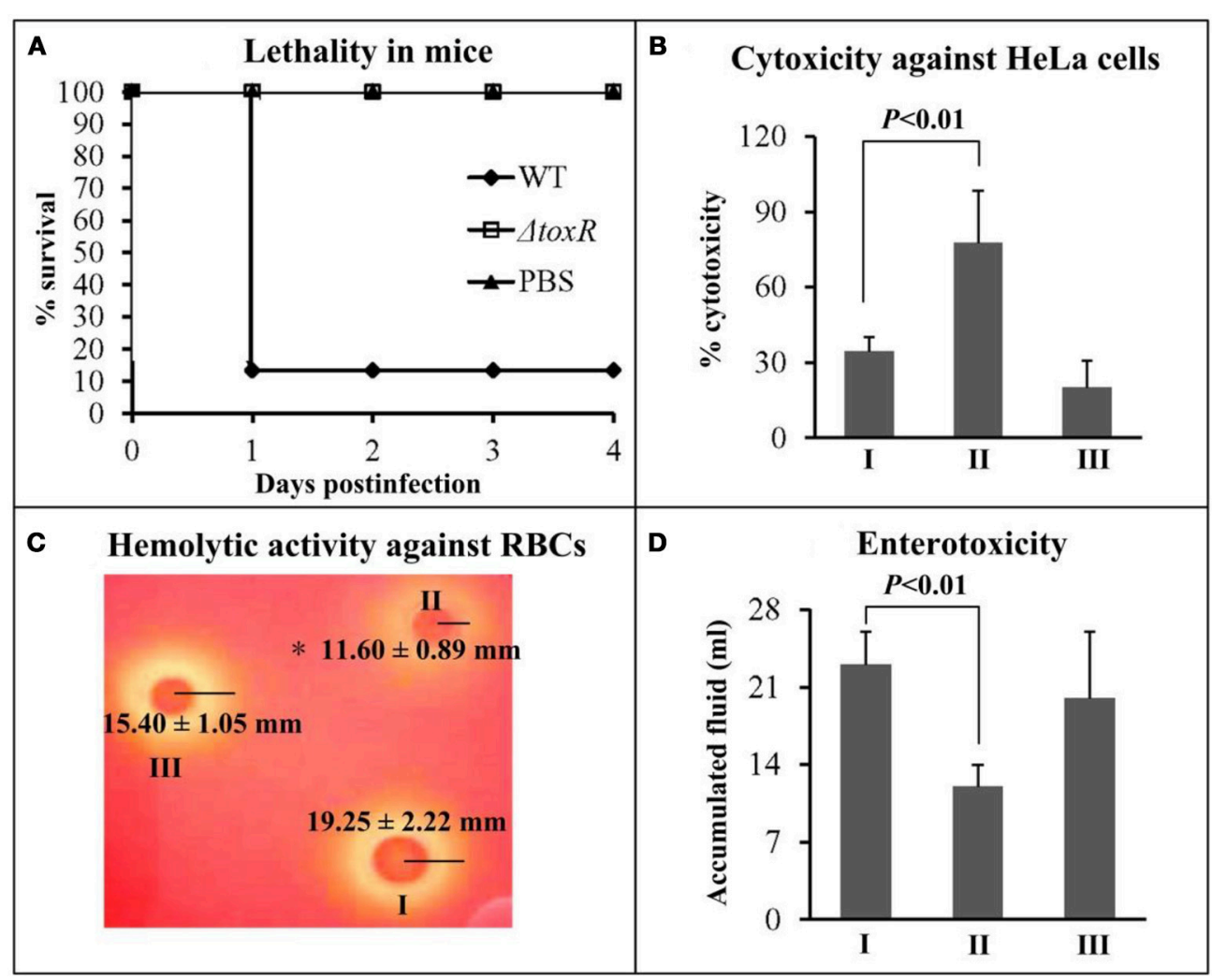

FIGURE 3 | Regulation of ToxR on virulence. I, II, and III represent WT/pBAD33, $\Delta$ toxR/pBAD33, and C- $\Delta$ toxR, respectively. (A) The mice survival rates infected with $V$. parahaemolyticus strains were measured to determine the lethality in mice. (B) The cytotoxicity against HeLa cells was evaluated in terms of the release of LDH.

(C) The hemolytic activity against RBCs was evaluated by measuring the radius of the $\beta$-hemolysin zone. Shown is a representative image of $V$. parahaemolyticus cells on Wagatsuma agar. Values are the mean \pm SD from three independent experiments with at least four replicates. The symbol ${ }^{*}$ represents $P<0.01$ for $\Delta$ toxR/pBAD33 vs. WT/pBAD33 or C- $\Delta$ toxR. (D) The enterotoxicity was evaluated by determining the fluid accumulation in the ileal loop.

agar (Figure 3C), and the hemolytic activity of $\Delta$ toxR/pBAD33 significantly decreased than that of WT/pBAD33 and C- $\Delta$ toxR. Finally, the enterotoxicity of $V$. parahaemolyticus strains were examined by using a rabbit ileal loop model (Figure 3D), and the results showed that the enterotoxicity of $\Delta$ toxR/pBAD33 significantly decreased in fluid accumulation compared with the $\mathrm{WT} / \mathrm{pBAD} 33$ and $\mathrm{C}-\Delta$ toxR. Taken together, ToxR inhibits the cytotoxicity, but it activates mouse lethality, hemolytic activity, and enterotoxicity of $V$. parahaemolyticus.

\section{Negative Regulation of T3SS1 by ToxR}

The first genes of putative operons exsBAD-vscBCD (VP17001688, T3SS1regulation), VP1687-1686 (T3SS1 effector), VP16671656 (T3SS1 apparatus) from T3SS1 locus were selected as the target genes (Table 2), and then subjected to primer extension, qPCR, LacZ fusion, EMSA, and DNase I footprinting assays. The primer extension assay (Figure 4A) detected a single transcriptional start site for each target gene, and the mRNA level of each target was enhanced in $\Delta$ toxR relative to WT. The qPCR (Figure 4B) further confirmed the mRNA levels of the three genes were enhanced in $\triangle$ toxR relative to WT. To test the action of ToxR on the promoter activity of the target genes, the recombinant lac $Z$ fusion plasmid that contains the indicated regulatory region and a promoterless lac $Z$ gene was transferred into $\Delta$ toxR and WT, respectively (Figure $4 \mathrm{C}$ ). The results disclosed a significantly increased promoter activity of each of the target genes tested in $\Delta$ tox $R$ relative to $\mathrm{WT}$. The promoter-proximal DNA regions of the above three genes were radioactively labeled, and subjected to EMSA with the purified His-ToxR (Figure 4D). His-ToxR was unable to bind to the upstream DNA fragment of exsB. However, it was able to bind to the upstream DNA fragments of VP1687 and VP1667. As further determined by DNA footprinting (Figure 4E), His-ToxR protected a single DNA region upstream of VP1687 and VP1667, respectively, against DNase I digestion, which were considered as the ToxR-binding sites. Taken together, ToxR indirectly represses the transcription of exs $B A D-v s c B C D$, while it negatively regulates the transcription of VP1687-1686 and VP1667-1656 in an indirect manner.

\section{Positive Regulation of Vp-PAI (T3SS2 and Tdh2) by ToxR}

The first genes of putative operons VPA1332-1333 (Vp-PAI regulation), VPA1362-1358 (T3SS2 apparatus), and $t$ dh2 from Vp-PAI locus were selected as the target genes (Table 2), and 


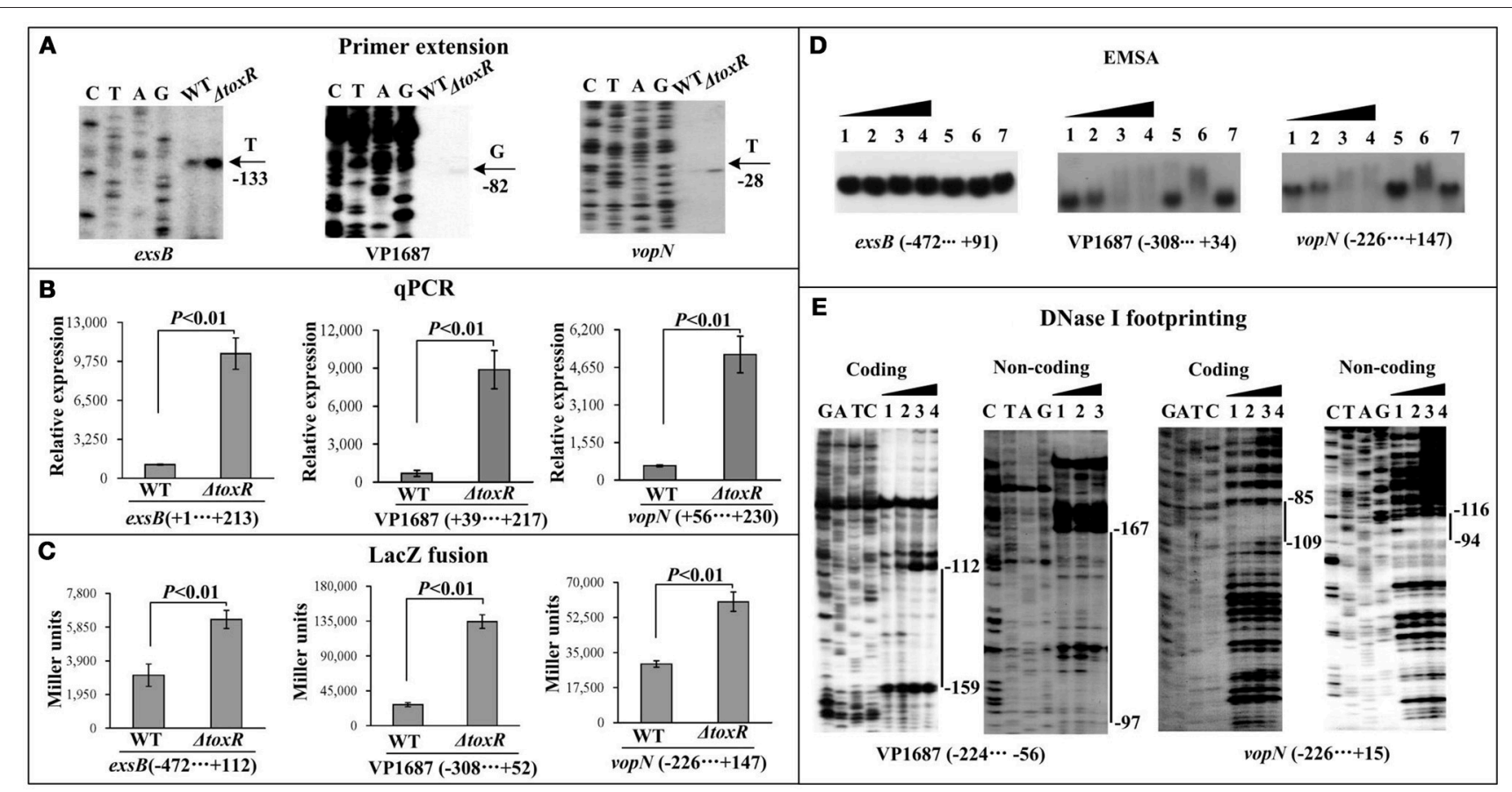

FIGURE 4 | Regulation of T3SS1 genes by ToxR. The primer extension (A) and GPCR (B) were done as Figure 1, the LacZ fusion (C) and EMSA (D) assays were done as Supplementary Figure 1, while the DNase I footprinting (E) was done as Figure 2.

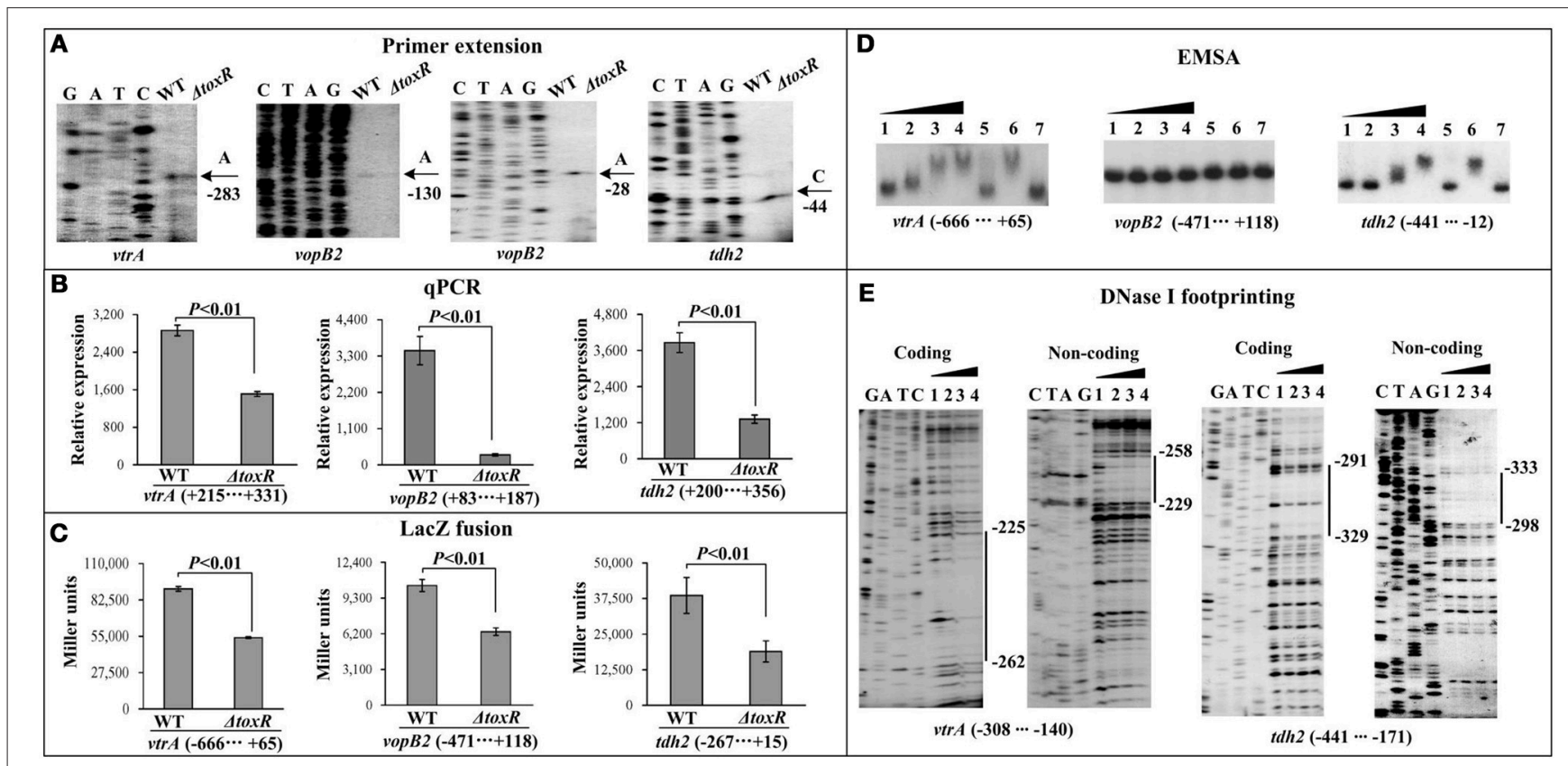

FIGURE 5 | Regulatory actions ToxR on Vp-PAl genes. The primer extension (A) and qPCR (B) were done as Figure 1, the LacZ fusion (C) and EMSA (D) assays were done as Supplementary Figure 1, while the DNase I footprinting (E) was done as Figure 2.

then subjected to the investigation of ToxR-mediated gene regulation via primer extension, $\mathrm{qPCR}$, LacZ fusion, EMSA, and DNase I footprinting assays. The primer extension and qPCR results showed that the transcription levels of all the three genes selected from $\mathrm{Vp}$-PAI decreased in $\Delta$ tox $\mathrm{R}$ relative to $\mathrm{WT}$ (Figures 5A,B). The lac $Z$ fusion results demonstrated that the promoter activity of each of the three operons in $\Delta$ toxR was much lower than that in WT (Figure 5C). As further determined 


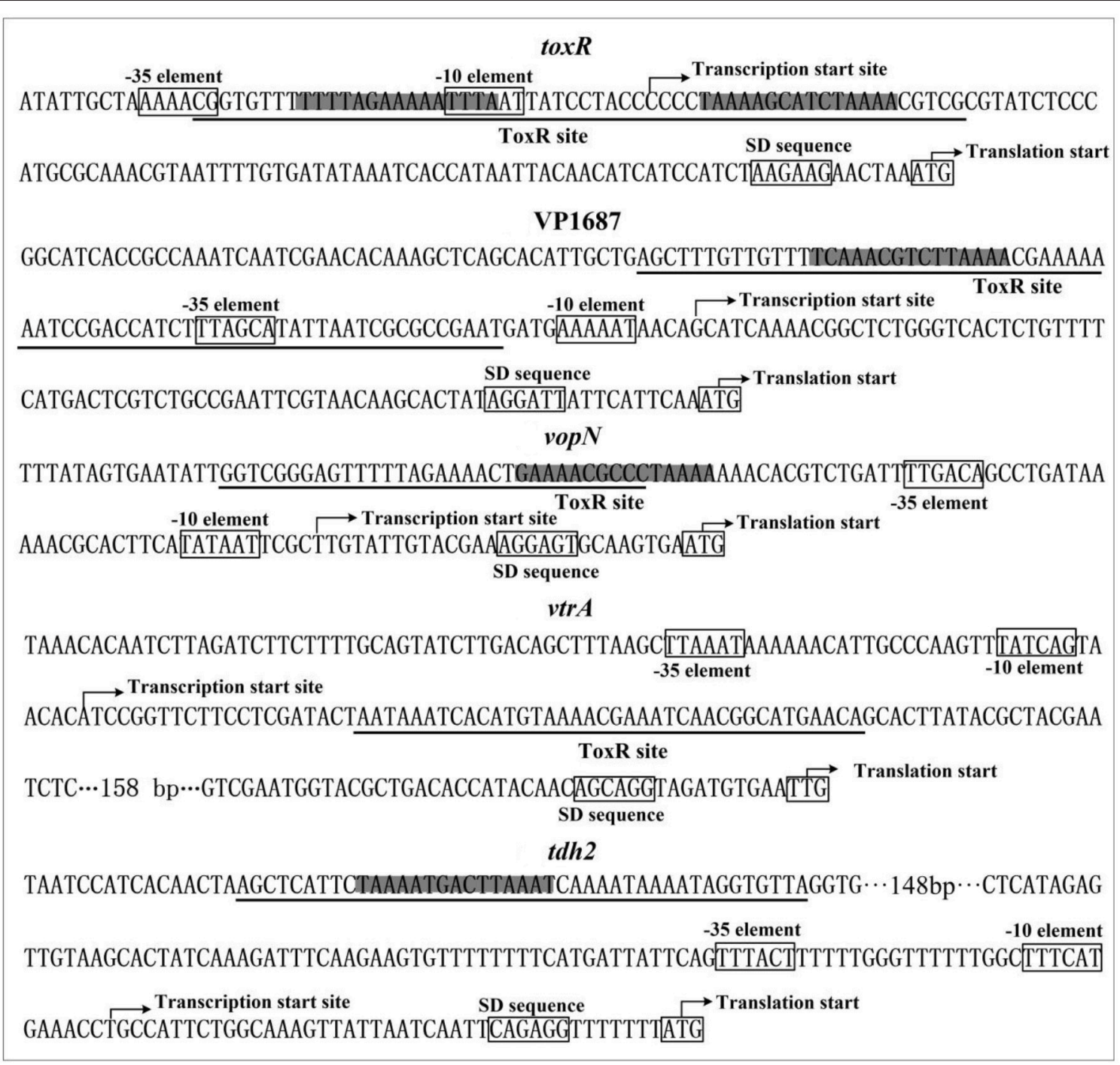

FIGURE 6 | Promoter structure of target genes. The promoter DNA regions of indicated genes were derived from RIMD 2210633. The translation and transcription starts were shown with bent arrows. The predicted core promoter -10 and -35 elements and the SD sequences were boxed. The ToxR box-like sequences were highlighted, and the ToxR sites were underlined.

by EMSA (Figure 5D), His-ToxR was able to specifically bind to the upstream DNA fragment of $v \operatorname{trA}$ (VPA1332) and $t d h 2$ in a dose-dependent manner, but a negative result was observed for vopB2 (VPA1362). The results of DNase I footprinting assay showed that His-ToxR protected a single DNA region upstream of $v t r A$ and $t d h 2$ against DNase I digestion, respectively, which were considered as the ToxR-binding sites (Figure 5E). Taken together, ToxR activates the transcription of VPA1332-1333 and $t d h 2$ in a direct manner, but it indirectly activates the transcription of VPA1362-1358.

\section{DISCUSSION}

A previous study showed that the transcriptional pattern of tox $R$ was consistent with that of $a p h A$, which was highly expressed at
$\mathrm{LCD}\left(\mathrm{OD}_{600}=0.05-0.2\right)$ when the bacteria were grown in Difco marine broth 2216 (BD Biosciences) at $37^{\circ} \mathrm{C}$ (Zhang et al., 2017). The phenomenon suggested a possible connection between toxR transcription and QS in V. parahaemolyticus. In the present work, we showed that the highest transcriptional level of toxR occurred at an $\mathrm{OD}_{600}=0.2-0.4$, but it dramatically decreased when the $\mathrm{OD}_{600}$ value was lower than 0.2 or higher than 0.4 , suggesting the transcriptional pattern of toxR depends on the bacterial growth conditions. We further investigated the regulatory actions of the QS regulators AphA and OpaR on toxR transcription. The results showed that AphA indirectly represses the transcription of toxR at LCD, whereas OpaR has no regulatory actions on tox $R$ transcription. Thus, there should be an additional unknown regulator (s) that contributes to the cell density-dependent transcriptional pattern of toxR. We previously showed that CalR 
occupies the regulatory region of tox $R$ to repress its transcription when the bacteria were harvested at the midlogarithmic growth phase (Osei-Adjei et al., 2017), and the expressional level of CalR increased considerably with the increase of cell density from an $\mathrm{OD}_{600}$ value of 0.05 to 1.2 (unpublished data). Moreover, we demonstrated herein that ToxR binds to its own promoterproximal DNA region to repress its own gene transcription at the transition from LCD to HCD $\left(\mathrm{OD}_{600}=0.2-0.4\right)$. Thus, the highest transcription of toxR occurs at an $\mathrm{OD}_{600}$ value of 0.2 to 0.4 and would be due to the subtle regulation of AphA, ToxR, and CalR. Nevertheless, whether there are additional unknown regulators that can regulate the transcription of tox $R$ during the growth periods of $V$. parahaemolyticus strain need to be further investigated.

The present work also demonstrated that ToxR acts as an inhibitor of the $V$. parahaemolyticus-induced cytotoxicity against HeLa cells, while it serves as an activator of the lethality in mice, the enterotoxicity in a rabbit ileal model, and the hemolytic activity against RBCs. Also, ToxR binds to the promoter-proximal DNA regions of VP1687-1686 and VP16671656 of the T3SS1 locus to repress their transcription, but it manifests indirect repression of exsBAD-vscBCD transcription. ToxR, also, occupies the promoter-proximal DNA regions of VPA1332-1333 and tdh2 of Vp-PAI locus to activate their transcription, but it only has indirect regulatory actions on VPA1362-1358 transcription. Thus, ToxR regulates the multiple virulence phenotypes via directly acting on the T3SS1 and VpPAI genes.

T3SS1 is under the subtle regulation of the transcriptional regulatory system ExsACDE in V. parahaemolyticus (Zhou et al., 2010; Erwin et al., 2012). ExsA acts as a transcriptional activator of T3SS1 genes (Zhou et al., 2010). ExsD interacts with ExsA to prevent the activation of ExsA (Zhou et al., 2010). ExsC binds ExsD to prevent the binding of ExsD to ExsA, allowing expression of T3SS1 gene (Zhou et al., 2010). ExsE can bind ExsC and, thereby, antagonizes ExsC activity (Erwin et al., 2012). Indirect regulation of exsBAD-vscBCD by ToxR indicates ToxR regulation of T3SS1 is not mediated by the ExsACDE system. However, CalR occupies the promoter-proximal DNA region of exs $B A D-v s c B C D$ to repress its transcription, and ToxR activates calR, and CalR feedback inhibits toxR and its own gene (OseiAdjei et al., 2017). Both ToxR and CalR bind to the promoterproximal DNA regions of one or more operons in T3SS locus to repress their transcription (Osei-Adjei et al., 2017). The operon VPA1332-1333 encodes the transcriptional activator VtrA of VpPAI; mutation of $v \operatorname{tr} A$ leads to reduced fluid accumulation in the rabbit intestine infection model (Kodama et al., 2010). The direct and positive regulatory action of ToxR on the transcription of $v \operatorname{tr} A$ indicates the entire $\mathrm{Vp}$-PAI genes are under the positive control of ToxR in $V$. parahaemolyticus.

\section{REFERENCES}

Broberg, C. A., Calder, T. J., and Orth, K. (2011). Vibrio parahaemolyticus cell biology and pathogenicity determinants. Microbes Infect. 13, 992-1001. doi: 10.1016/j.micinf.2011.06.013
The organization of toxR, VP1687-1686, VP1667-1656, VPA1332-1333, and $t d h 2$ promoters were reconstructed herein, by collecting the data of translation/transcription start sites, the core promoter -10 and -35 elements, ToxR binding sites, ToxR box-like sequences, ribosomal binding Shine-Dalgarno (SD) sequences (Figure 6). The ToxR-binding sites for both toxR and VP1687-1686 overlap the core -35 and/or -10 elements, and thus ToxR is thought to silence the transcription of toxR and VP1687 - 1686 by directly interfering with RNA polymerase (RNAP) action. The ToxR binding site for VP1667-1656 was detected in the upstream of the -35 element, which is unusual for a regulator that represses its target gene transcription. The binding site of ToxR to $v \operatorname{tr} A$ promoter is located downstream of the transcription start site, which is also unusual for a regulator that stimulates its target gene transcription. However, similar regulatory mechanisms have been observed in other species, such as S. enterica (Shi et al., 2004), E. coli (Munson and Scott, 2000), and Y. pestis (Zhang et al., 2013). The ToxR-binding site for $t d h 2$ was located upstream of the promoter -35 element. Thus, the ToxR-stimulated tdh 2 promoter may have a class I transcriptional stimulation that depends on the subunit Cterminal domain of RNAP to function (Ishihama, 2000). It should be noted that not all ToxR-binding sites contain the ToxR boxlike sequences, indicating the computational analysis is not a panacea.

Taken together, the present work reports of the autoregulation of ToxR and its regulatory actions on major virulence gene loci in $V$. parahaemolyticus, which is beneficial to the pathogenesis of the pathogen. The data presented here also provide vital information for a deeper understanding of the regulatory patterns of ToxR in V. parahaemolyticus.

\section{AUTHOR CONTRIBUTIONS}

$\mathrm{DZ}, \mathrm{XH}$, and RY conceived the study and designed experimental procedures. YiqZ, LH, YinZ, WY, ZY, RL, XS performed the experiments and carried out data analysis. YiqZ, GO-A, DZ, and $\mathrm{XH}$ wrote the paper.

\section{FUNDING}

This work was supported by the National Natural Science Foundation of China (81601809, and 31671290).

\section{SUPPLEMENTARY MATERIAL}

The Supplementary Material for this article can be found online at: https://www.frontiersin.org/articles/10.3389/fcimb. 2018.00291/full\#supplementary-material

Childers, B. M., and Klose, K. E. (2007). Regulation of virulence in Vibrio cholerae: the ToxR regulon. Future Microbiol. 2, 335-344. doi: 10.2217/17460913. 2.3.335

Crawford, J. A., Krukonis, E. S., and Dirita, V. J. (2003). Membrane localization of the ToxR winged-helix domain is required for TcpP-mediated 
virulence gene activation in Vibrio cholerae. Mol. Microbiol. 47, 1459-1473. doi: 10.1046/j.1365-2958.2003.03398.x

Dirita, V. J., Parsot, C., Jander, G., and Mekalanos, J. J. (1991). Regulatory cascade controls virulence in Vibrio cholerae. Proc. Natl. Acad. Sci. U.S.A. 88, 5403-5407. doi: 10.1073/pnas.88.12.5403

Erwin, D. P., Nydam, S. D., and Call, D. R. (2012). Vibrio parahaemolyticus ExsE is requisite for initial adhesion and subsequent type III secretion system 1-dependent autophagy in HeLa cells. Microbiology 158(Pt 9): 2303-2314. doi: 10.1099/mic.0.059931-0

Gao, H., Zhang, Y., Yang, L., Liu, X., Guo, Z., Tan, Y., et al. (2011). Regulatory effects of cAMP receptor protein (CRP) on porin genes and its own gene in Yersinia pestis. BMC Microbiol. 11:40. doi: 10.1186/1471-2180-11-40

Goss, T. J., Morgan, S. J., French, E. L., and Krukonis, E. S. (2013). ToxR recognizes a direct repeat element in the toxT, ompU, ompT, and $c t x A$ promoters of Vibrio cholerae to regulate transcription. Infect. Immun. 81, 884-895. doi: 10.1128/IAI.00889-12

Higgins, D. E., and Dirita, V. J. (1994). Transcriptional control of toxT, a regulatory gene in the ToxR regulon of Vibrio cholerae. Mol. Microbiol. 14, 17-29. doi: 10.1111/j.1365-2958.1994.tb01263.x

Hiyoshi, H., Kodama, T., Iida, T., and Honda, T. (2010). Contribution of Vibrio parahaemolyticus virulence factors to cytotoxicity, enterotoxicity, and lethality in mice. Infect. Immun. 78, 1772-1780. doi: 10.1128/IAI.01051-09

Honda, T., Chearskul, S., Takeda, Y., and Miwatani, T. (1980). Immunological methods for detection of Kanagawa phenomenon of Vibrio parahaemolyticus. J. Clin. Microbiol. 11, 600-603.

Honda, T., Ni, Y. X., and Miwatani, T. (1988). Purification and characterization of a hemolysin produced by a clinical isolate of Kanagawa phenomenon-negative Vibrio parahaemolyticus and related to the thermostable direct hemolysin. Infect. Immun. 56, 961-965.

Hubbard, T. P., Chao, M. C., Abel, S., Blondel, C. J., Abel Zur Wiesch, P., Zhou, X., et al. (2016). Genetic analysis of Vibrio parahaemolyticus intestinal colonization. Proc. Natl. Acad. Sci. U.S.A. 113, 6283-6288. doi: $10.1073 /$ pnas. 1601718113

Hung, D. T., and Mekalanos, J. J. (2005). Bile acids induce cholera toxin expression in Vibrio cholerae in a ToxT-independent manner. Proc. Natl. Acad. Sci. U.S.A. 102, 3028-3033. doi: 10.1073/pnas.0409559102

Ishihama, A. (2000). Functional modulation of Escherichia coli RNA polymerase. Annu. Rev. Microbiol. 54, 499-518. doi: 10.1146/annurev.micro.54.1.499

Kleber-Janke, T., and Becker, W. M. (2000). Use of modified BL21(DE3) Escherichia coli cells for high-level expression of recombinant peanut allergens affected by poor codon usage. Protein Expr. Purif. 19, 419-424. doi: $10.1006 /$ prep.2000.1265

Kodama, T., Gotoh, K., Hiyoshi, H., Morita, M., Izutsu, K., Akeda, Y., et al. (2010). Two regulators of Vibrio parahaemolyticus play important roles in enterotoxicity by controlling the expression of genes in the Vp-PAI region. PLoS ONE 5:e8678. doi: 10.1371/journal.pone.0008678

Letchumanan, V., Chan, K. G., and Lee, L. H. (2014). Vibrio parahaemolyticus: a review on the pathogenesis, prevalence, and advance molecular identification techniques. Front. Microbiol. 5:705. doi: 10.3389/fmicb.2014.00705

Lin, Z., Kumagai, K., Baba, K., Mekalanos, J. J., and Nishibuchi, M. (1993). Vibrio parahaemolyticus has a homolog of the Vibrio cholerae toxRS operon that mediates environmentally induced regulation of the thermostable direct hemolysin gene. J. Bacteriol. 175:3844. doi: 10.1128/jb.175.12.3844-3855.1993

Lu, R., Osei-Adjei, G., Huang, X., and Zhang, Y. (2018). Role and regulation of the orphan AphA protein of quorum sensing in pathogenic Vibrios. Future Microbiol. 13, 383-391. doi: 10.2217/fmb-2017-0165

Makino, K., Oshima, K., Kurokawa, K., Yokoyama, K., Uda, T., Tagomori, K., et al. (2003). Genome sequence of Vibrio parahaemolyticus: a pathogenic mechanism distinct from that of $V$ cholerae. Lancet 361, 743-749. doi: 10.1016/S0140-6736(03)12659-1

Miyamoto, Y., Kato, T., Obara, Y., Akiyama, S., Takizawa, K., and Yamai, S. (1969). In vitro hemolytic characteristic of Vibrio parahaemolyticus: its close correlation with human pathogenicity. J. Bacteriol. 100, 1147-1149.

Munson, G. P., and Scott, J. R. (2000). Rns, a virulence regulator within the AraC family, requires binding sites upstream and downstream of its own promoter to function as an activator. Mol. Microbiol. 36, 1391-1402. doi: $10.1046 / j .1365-2958.2000 .01957 . x$
Naim, R., Yanagihara, I., Iida, T., and Honda, T. (2001). Vibrio parahaemolyticus thermostable direct hemolysin can induce an apoptotic cell death in Rat-1 cells from inside and outside of the cells. FEMS Microbiol. Lett. 195, 237-244. doi: 10.1111/j.1574-6968.2001.tb10527.x

Nair, G. B., Ramamurthy, T., Bhattacharya, S. K., Dutta, B., Takeda, Y., and Sack, D. A. (2007). Global dissemination of Vibrio parahaemolyticus serotype O3:K6 and its serovariants. Clin. Microbiol. Rev. 20, 39-48. doi: 10.1128/CMR. 00025-06

Ng, W. L., and Bassler, B. L. (2009). Bacterial quorum-sensing network architectures. Annu. Rev. Genet. 43, 197-222. doi: 10.1146/annurev-genet-102108-134304

Nishibuchi, M., Fasano, A., Russell, R. G., and Kaper, J. B. (1992). Enterotoxigenicity of Vibrio parahaemolyticus with and without genes encoding thermostable direct hemolysin. Infect. Immun. 60, 3539-3545.

Nishibuchi, M., and Kaper, J. B. (1990). Duplication and variation of the thermostable direct haemolysin (tdh) gene in Vibrio parahaemolyticus. Mol. Microbiol. 4, 87-99. doi: 10.1111/j.1365-2958.1990.tb0 2017.x

Okuda, J., and Nishibuchi, M. (1998). Manifestation of the Kanagawa phenomenon, the virulence-associated phenotype, of Vibrio parahaemolyticus depends on a particular single base change in the promoter of the thermostable direct haemolysin gene. Mol. Microbiol. 30, 499-511. doi: 10.1046/j.1365-2958.1998.01072.x

Osei-Adjei, G., Gao, H., Zhang, Y., Zhang, L., Yang, W., Yang, H., et al. (2017). Regulatory actions of ToxR and CalR on their own genes and type III secretion system 1 in Vibrio parahaemolyticus. Oncotarget 8, 65809-65822. doi: 10.18632/oncotarget.19498

Parales, R. E., and Harwood, C. S. (1993). Construction and use of a new broadhost-range lac $Z$ transcriptional fusion vector, pHRP309, for gram- bacteria. Gene 133, 23-30. doi: 10.1016/0378-1119(93)90220-W

Park, K. S., Ono, T., Rokuda, M., Jang, M. H., Iida, T., and Honda, T. (2004a). Cytotoxicity and enterotoxicity of the thermostable direct hemolysindeletion mutants of Vibrio parahaemolyticus. Microbiol. Immunol. 48, 313-318. doi: 10.1111/j.1348-0421.2004.tb03512.x

Park, K. S., Ono, T., Rokuda, M., Jang, M. H., Okada, K., Iida, T., et al. (2004b). Functional characterization of two type III secretion systems of Vibrio parahaemolyticus. Infect. Immun. 72, 6659-6665. doi: 10.1128/IAI.72.11.6659-6665.2004

Provenzano, D., and Klose, K. E. (2000). Altered expression of the ToxR-regulated porins $\mathrm{OmpU}$ and OmpT diminishes Vibrio cholerae bile resistance, virulence factor expression, and intestinal colonization. Proc. Natl. Acad. Sci. U.S.A. 97, 10220-10224. doi: 10.1073/pnas.170219997

Provenzano, D., Lauriano, C. M., and Klose, K. E. (2001). Characterization of the role of the ToxR-modulated outer membrane porins OmpU and OmpT in Vibrio cholerae virulence. J. Bacteriol. 183, 3652-3662. doi: 10.1128/JB.183.12.3652-3662.2001

Raimondi, F., Kao, J. P., Fiorentini, C., Fabbri, A., Donelli, G., Gasparini, N., et al. (2000). Enterotoxicity and cytotoxicity of Vibrio parahaemolyticus thermostable direct hemolysin in in vitro systems. Infect. Immun. 68, 3180-3185. doi: 10.1128/IAI.68.6.3180-3185.2000

Shi, Y., Latifi, T., Cromie, M. J., and Groisman, E. A. (2004). Transcriptional control of the antimicrobial peptide resistance ugtL gene by the Salmonella PhoP and SlyA regulatory proteins. J. Biol. Chem. 279, 38618-38625. doi: 10.1074/jbc.M406149200

Srivastava, D., and Waters, C. M. (2012). A tangled web: regulatory connections between quorum sensing and cyclic Di-GMP. J. Bacteriol. 194, 4485-4493. doi: 10.1128/JB.00379-12

Sun, F., Zhang, Y., Qiu, Y., Yang, H., Yang, W., Yin, Z., et al. (2014). H-NS is a repressor of major virulence gene loci in Vibrio parahaemolyticus. Front. Microbiol. 5:675. doi: 10.3389/fmicb.2014.00675

Sun, F., Zhang, Y., Wang, L., Yan, X., Tan, Y., Guo, Z., et al. (2012). Molecular characterization of direct target genes and cis-acting consensus recognized by quorum-sensing regulator AphA in Vibrio parahaemolyticus. PLoS ONE 7:e44210. doi: 10.1371/journal.pone.0044210

Thompson, F. L., Iida, T., and Swings, J. (2004). Biodiversity of vibrios. Microbiol. Mol. Biol. Rev. 68, 403-431. doi: 10.1128/MMBR.68.3.403-431.2004 
Valeru, S. P., Wai, S. N., Saeed, A., Sandström, G., and Abd, H. (2012). ToxR of Vibrio cholerae affects biofilm, rugosity and survival with Acanthamoeba castellanii. BMC Res. Notes 5:33. doi: 10.1186/1756-0500-5-33

Van Helden, J. (2003). Regulatory sequence analysis tools. Nucleic Acids Res. 31, 3593-3596. doi: 10.1093/nar/gkg567

Whitaker, W. B., Parent, M. A., Boyd, A., Richards, G. P., and Boyd, E. F. (2012). The Vibrio parahaemolyticus ToxRS regulator is required for stress tolerance and colonization in a novel orogastric streptomycin-induced adult murine model. Infect. Immun. 80, 1834-1845. doi: 10.1128/IAI. 06284-11

Xu, X., Stern, A. M., Liu, Z., Kan, B., and Zhu, J. (2010). Virulence regulator AphB enhances toxR transcription in Vibrio cholerae. BMC Microbiol. 10:3. doi: $10.1186 / 1471-2180-10-3$

Yeung, P. S., and Boor, K. J. (2004). Epidemiology, pathogenesis, and prevention of foodborne Vibrio parahaemolyticus infections. Foodborne Pathog. Dis. 1, 4-88. doi: 10.1089/153531404323143594

Zhang, Y., Gao, H., Osei-Adjei, G., Zhang, Y., Yang, W., Yang, H., et al. (2017). Transcriptional regulation of the type VI secretion system 1 genes by quorum sensing and ToxR in Vibrio parahaemolyticus. Front. Microbiol. 8:2005. doi: 10.3389/fmicb.2017.02005

Zhang, Y., Qiu, Y., Tan, Y., Guo, Z., Yang, R., and Zhou, D. (2012). Transcriptional regulation of $o p a R, q r r 2-4$ and $a p h A$ by the master quorumsensing regulator OpaR in Vibrio parahaemolyticus. PLoS ONE 7:e34622. doi: $10.1371 /$ journal.pone.0034622
Zhang, Y., Wang, L., Han, Y., Yan, Y., Tan, Y., Zhou, L., et al. (2013). Autoregulation of PhoP/PhoQ and positive regulation of the cyclic AMP receptor protein-cyclic AMP complex by PhoP in Yersinia pestis. J. Bacteriol. 195, 1022-1030. doi: 10.1128/JB.01530-12

Zhou, D., Yan, X., Qu, F., Wang, L., Zhang, Y., Hou, J., et al. (2013). Quorum sensing modulates transcription of $c p s Q-m f p A B C$ and $m f p A B C$ in Vibrio parahaemolyticus. Int. J. Food Microbiol. 166, 458-463. doi: 10.1016/j.ijfoodmicro.2013.07.008

Zhou, X., Konkel, M. E., and Call, D. R. (2010). Regulation of type III secretion system 1 gene expression in Vibrio parahaemolyticus is dependent on interactions between ExsA, ExsC, and ExsD. Virulence 1, 260-272. doi: 10.4161/viru.1.4.12318

Conflict of Interest Statement: The authors declare that the research was conducted in the absence of any commercial or financial relationships that could be construed as a potential conflict of interest.

Copyright (C) 2018 Zhang, Hu, Osei-Adjei, Zhang, Yang, Yin, Lu, Sheng, Yang, Huang and Zhou. This is an open-access article distributed under the terms of the Creative Commons Attribution License (CC BY). The use, distribution or reproduction in other forums is permitted, provided the original author(s) and the copyright owner(s) are credited and that the original publication in this journal is cited, in accordance with accepted academic practice. No use, distribution or reproduction is permitted which does not comply with these terms. 